\title{
Effects of Friction Surfacing on the Characteristics of Consumable Rods of Ti-6Al-4V
}

\author{
Natalia do Vale ${ }^{a} \oplus^{\circledR}$,Viktoria Fitseva ${ }^{b}$, Stefanie Hanke ${ }^{b}$, Severino Leopoldino Urtiga Filho ${ }^{a}$, \\ Jorge Fernandez dos Santos ${ }^{b}$

\begin{abstract}
${ }^{a}$ Departamento de Engenharia Mecânica, Universidade Federal de Pernambuco, Recife, PE, Brasil ${ }^{b}$ Helmholtz-Zentrum Geesthacht GmbH, Institute of Materials Research, Materials Mechanics, Solid-State Joining Processes, Geesthacht, Germany
\end{abstract}

Received: December 13, 2018; Revised: September 05, 2019; Accepted: January 12, 2020

Friction surfacing (FS) is a coating technique applied mainly in corrosion protection and repair of components. The study addresses the effects of deposition and rotational speeds on the rods characteristics and process efficiency for the deposition of Ti-6Al-4V on self-mating substrates by FS. The consumption rate was $1.8 \mathrm{~mm} / \mathrm{s}$, deposition speeds of 8,16 and $24 \mathrm{~mm} / \mathrm{s}$ and rotational speeds of 2000,3000 and $4000 \mathrm{rpm}$. It was shown that the flash forms primarily at the rod, ascending around the tip and leaving the coating without flash. The higher deposition speeds led to a decrease in rod thickness and diameter. For higher rotational speeds, an increase in diameter and decrease in thickness is observed for the flash on the rod. Experiments have shown that the rotational and deposition speeds have a decisive influence on the flash formation. Its microstructure changes due to the welding process and a change in hardness can be observed.

Keywords: Friction surfacing, titanium, consumable rod.

\section{Introduction}

The Friction Surfacing (FS) technique is a coating process mainly used with the purpose of extending the service life of various components by depositing mostly metallic materials in a solid state. The deposition is generally used to repair worn parts and improve the wear resistance and anticorrosion properties, being considered as a process for local repair. Although FS was first patented in 1941, by Klopstock and Neelands, only about the late 80 s it has been studied more frequently with industries' applications.

FS is a welding process based on the plastic deformation of a rotating consumable rod by the frictional heat generated in the interface between rod and substrate. As a solid state process, the coatings are generated at temperatures below the melting range of the materials. A schematic presentation of the process is shown in Fig. 1.

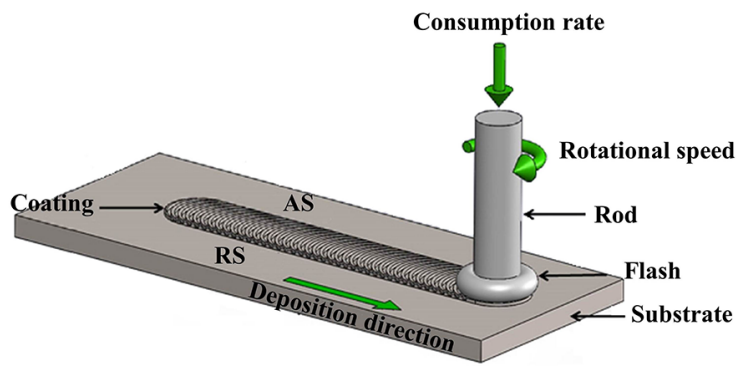

Figure 1. Schematic of $\mathrm{FS}^{1}$.
The length of a single deposit is limited by the dimension of the consumable rod. During the deposition process, generally a revolving flash of plasticized material is generated, ascending around the rod and leaving the coating without flash. The flash is formed as a consequence of a portion of the plasticized material from the periphery of the rods being squeezed out of the contact zone without lateral confinement and pushed upwards around the rod by the axial force, giving it a mushroom-shaped geometry ${ }^{1,2,3,4}$. Hence, the size of the flash moving up towards the rod limits even further the length of a deposit. Flash plays an important role as boundary condition of temperature and pressure for the joining process 5 .

The process parameters influence the deposition efficiency, which is the ratio of metal deposited to total consumed metal, directly related to the amount of flash generated. For example, according to Li and Shinoda $(2000)^{6}$, an increase in axial force leads to a reduction of deposition efficiency due to the higher amount of flash generated. Previous studies on Ti Grade 1 depositions ${ }^{7}$ have shown that for lower rotational speeds (2000 rpm), non-homogeneous deposition surfaces were obtained, presenting a considerable amount of material being deposited irregularly along the retreating side of the coating. Flash formation on Ti-6Al-4V and Ti Grade 1 were also found in Fitseva's et. al. study (2016) ${ }^{8}$, but for the first it was found in a range of 300-1000 rpm and for the second about 300-2000 rpm. The larger extension of flash on Ti Grade 1 may be directly related to the lack of alloying elements in its composition, presenting lower resistance and leading to higher temperatures ${ }^{8}$. 
The feasibility of Ti-6Al-4V coatings on self-mating substrates was shown recently ${ }^{1,8,9}$ and the obtained results were successful. Titanium is an expensive material, though it has interesting properties and its deposition can be used in a wide range of applications. Therefore, it is necessary to understand the flash formation on titanium in order to reduce the material being lost and optimize the process.

In the present study, the FS process was applied to Ti-6Al-4V on a self-mating substrate. The flash formation, deposition efficiency and rods geometry and properties are investigated. This study has been developed with the purpose of investigating the effects of rotational and deposition speeds on the consumable rods after the deposition process, in order to understand the process efficiency by loss of material in flash formation on the rod.

\section{Experimental Procedure}

FS was performed in a custom-built friction welding machine, shown by Vale et. al. ${ }^{9}$ and Hanke et. al. ${ }^{10}$, capable of delivering $8 \mathrm{kN}$ axial force and $60 \mathrm{Nm}$ of torque, and was equipped with $20 \mathrm{~mm}$ diameter Ti-6Al-4V rods with approximately $150 \mathrm{~mm}$ length. Ti-6A-4V $2 \mathrm{~mm}$ thick was used as substrate. Chemical compositions as given by the supplier are shown in Table 1.

The process parameters selected were the rod consumption rate, the deposition speed and the rotational speed. Rods were fed into the process zone at a constant axial speed of $1.8 \mathrm{~mm} / \mathrm{s}$, the deposition speeds varied between 8,16 and $24 \mathrm{~mm} / \mathrm{s}$ and the rotational speeds used were 2000, 3000 and $4000 \mathrm{rpm}$. Cross sections of coatings and rods analysis have been prepared according to standard metallographic procedures (ASTM E3-0118). Optical microscope was used to evaluate the rods geometry and image processing techniques were used for the measurements. Microhardess profiles along the longitudinal cross sections of coating and rod were conducted by Vickers hardness, using $0.2 \mathrm{~N}$ for 10 s, according to the standard ASTM E 384-10.

\section{Results and Discussion}

\subsection{Flash formation}

During the FS process, the material is highly plasticized at the tip of the consumable rod and is pressed against the substrate without lateral confinement, developing a flash and a lack of bonding at the coating edges on both advancing (AS) and retreating sides (RS) ${ }^{11}$.
The majority of the conditions resulted in a coating with a homogeneous surface, without flash formation, as can be seen in Fig. 2 (b-j). Although, when applying the lowest rotational speed $(2000 \mathrm{rpm})$ it could be noticed an accumulation of material on the RS of the coating (Fig, 2 (a)). In spite of, even for this condition the flash ascended around the rod in a mushroom-shaped geometry (Fig. 2 (b)).

The majority of the publications, on various materials processed by FS, do not report the flash formation at the coating. Although, recently Fitseva et. al. ${ }^{1}$ registered the possibility of flash formation on Ti-6Al-4V coatings due to the wide range of rotational speeds applied on that study. The referred study showed that, for titanium coatings, under low rotational speeds (300, 400 and $600 \mathrm{rpm})$, the flash was deposited along the RS of the layer leaving the rod tip without flash. For the deposition of Ti Grade 1 on Ti-6Al-4V, Vale et. al. ${ }^{7}$ revealed that a rotational speed of $2000 \mathrm{rpm}$ resulted in a coating with an inhomogeneous layer appearance, presenting a large amount of material being deposited along the RS. In spite of this tendency for material accumulation at the RS, for those experiments the flash was formed at the consumable rod. Then, it can be expected that when reducing the rotational speed below $2000 \mathrm{rpm}$ for the deposition of Ti-6Al-4V under the conditions of the present study, flash formation at the RS of the coatings may arise.

The energy input is considered as an important parameter for understanding the flash formation behavior. Previous studies have assumed that the material transferred into the flash and the deposited material do not undergo the same amount of deformation ${ }^{4}$. In this way, it can be stated that they do not experience the same energy input. Nevertheless, the energy input per unit length equation (Eq. 1), derived from the friction stir welding process, can be used to establish a correlation with the flash formation at the coating ${ }^{12}$ :

$$
E I=2 \pi T \Omega / \nu
$$

where $\mathrm{T}$ is the torque $[\mathrm{Nm}], \Omega$ is the rotational speed $[\mathrm{rpm}]$ and $v$ is the deposition speed $[\mathrm{mm} / \mathrm{s}]$. Therefore, the rotational speed of the rod and the deposition speed have an important influence on the flash formation. The energy input per unit length needs to be lowered in order to form the flash on the rod instead of on the coating. Thus, to avoid flash formation at the coating for low rotational speeds, the deposition speed needs to be increased in order to decrease the energy input.

Table 1. Chemical composition (in weight-\%).

\begin{tabular}{ccccccccccc}
\hline & Material & Fe & $\mathbf{C}$ & $\mathbf{N}$ & $\mathbf{H}$ & $\mathbf{O}$ & $\mathbf{A l}$ & $\mathbf{V}$ & $\mathbf{T i}$ \\
\hline Rod 1 & Ti-6Al-4V & 0.11 & 0.03 & 0.023 & 0.0039 & 0.15 & 6.6 & 3.5 & Bal. \\
Substrate & Ti-6Al-4V & 0.11 & 0.02 & 0.007 & 0.0049 & 0.049 & 6.2 & 3.9 & Bal. \\
\hline
\end{tabular}




\section{$\stackrel{\text { Deposition direction }}{\longrightarrow}$}

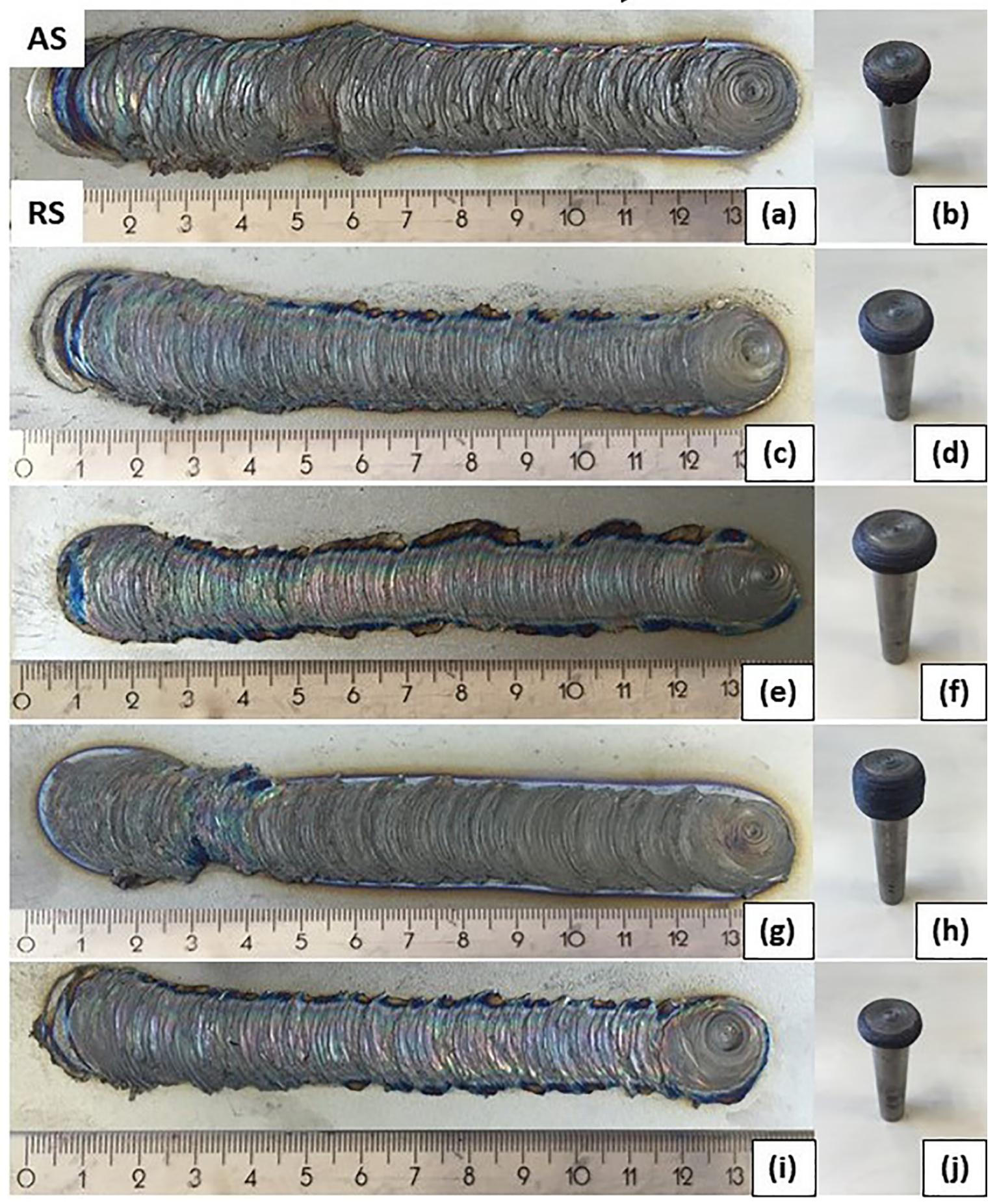

Figure 2. Coating surface appearance deposited and consumable rod after the FS process for (a-b) $16 \mathrm{~mm} / \mathrm{s}$ and $2000 \mathrm{rpm}$, (c-d) $16 \mathrm{~mm} / \mathrm{s}$ and $3000 \mathrm{rpm}$, (e-f) $16 \mathrm{~mm} / \mathrm{s}$ and $4000 \mathrm{rpm}$, (g-h) $8 \mathrm{~mm} / \mathrm{s}$ and $3000 \mathrm{rpm}$ and (i-j) $24 \mathrm{~mm} / \mathrm{s}$ and $3000 \mathrm{rpm}$.

\subsection{Deposition efficiency}

Reducing the amount of material being lost in the flash extend the efficiency of the process. The generation of a revolving flash at the consumable rod tip contributes to a decrease in mass transfer efficiency, as it represents a fraction of material that does not bond to the substrate ${ }^{11,13}$. Since the maximum length of the coating to be deposited is determined by the rod length, the coating length may be reduced by the amount of material being lost on flash formation. 
The deposition efficiency $(\eta)$ is the relationship between the amount of deposited material $\left(\mathrm{V}_{\text {deposited }}\right)$ and the consumed rod volume $\left(\mathrm{V}_{\text {consumed }}\right)$, revealing the portion of the processed rod material that was deposited, and which was transferred to the flash. Then, the deposition efficiency can be calculated by ${ }^{1,4}$ :

$\eta_{\text {deposition }}=\frac{V \text { deposited }}{V \text { consumed }} x 100 \%=\frac{d x w x v_{x}}{\pi x r^{2} x v_{c r}} x 100 \%$ Eq. 2

where $d$ is the layer thickness, $w$ is the total coating width, $v_{\mathrm{x}}$ is the deposition speed, $r$ is the consumable rod radius $(10 \mathrm{~mm})$ and $v_{\text {cr }}$ is the rod consumption rate. The cross section area of the coating without flash is used, because flash cannot be considered as effective deposition.

Due to the formation of unbonded regions on the coatings edges, just a portion of the deposited material is effectively joined. Therefore, the joining efficiency is given by the ratio between the bonded width $\left(W_{b}\right)$ and the maximum coating length $\left(W_{d}\right)^{5}$ :

$$
\eta_{\text {joining }}=\frac{W_{b}}{W_{d}}
$$

Thus, effective coating efficiency reflects the fraction of consumed rod that is actually bonded to the substrate and is estimated by:

$$
\eta_{\text {coating }}=\eta_{\text {deposition }} x \eta_{\text {joining }}
$$

Eq. 4
Fig. 3 presents the effects of process parameters on the coating efficiency. Depending on the rotational speed, the coating efficiency varied between $22 \%$ and $34 \%$. In relation to the deposition speed, the variation was from $19 \%$ to $25 \%$. As described by Gandra's et. al. ${ }^{5}$, the effect of rotational speed is more pronounced, while the coating efficiency is not significantly affected by the deposition speed. The correlation of coating efficiency and rotational speed was not linear, while by increasing the deposition speed it led to a slightly increase in the coating efficiency. On Fitseva's et al. ${ }^{1}$ study on Ti-6Al-4V the deposition efficiency varied between $12 \%$ and $39 \%$ depending on the rotational speed. The lower rotational speeds applied on that study provided minor deposition efficiencies.

\subsection{Characteristics of the rods}

No publications could be found so far in the literature about the surface characteristics of the rods after the deposition. Although the rods characteristics are not an usual parameter considered for the process, its understanding allows the increasing of process efficiency. Fig. 4 shows that the flash formation ascending on the rod is dependent on the process parameters. The flash diameter increases and its thickness slightly decreases by enhancing the rotational speed. This is probably due to the fact that higher rotational speeds implies in higher centrifugal forces, and the diameter tends to get larger.
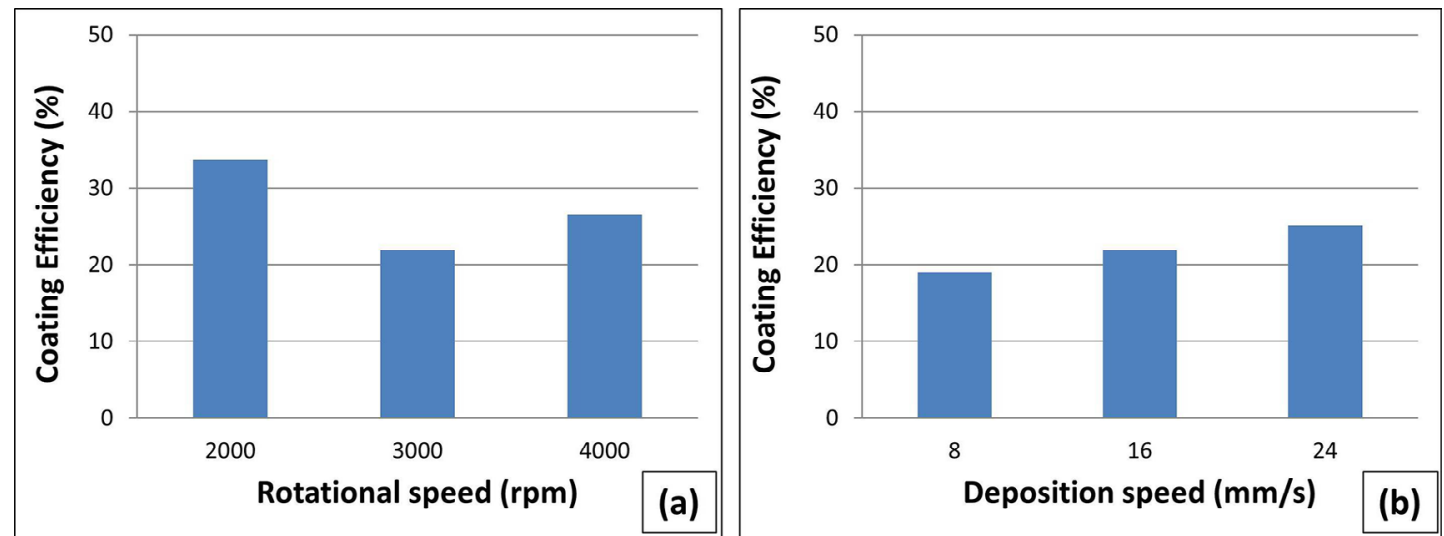

Figure 3. Coating efficiency as a function of the (a) rotational and (a) deposition speeds.
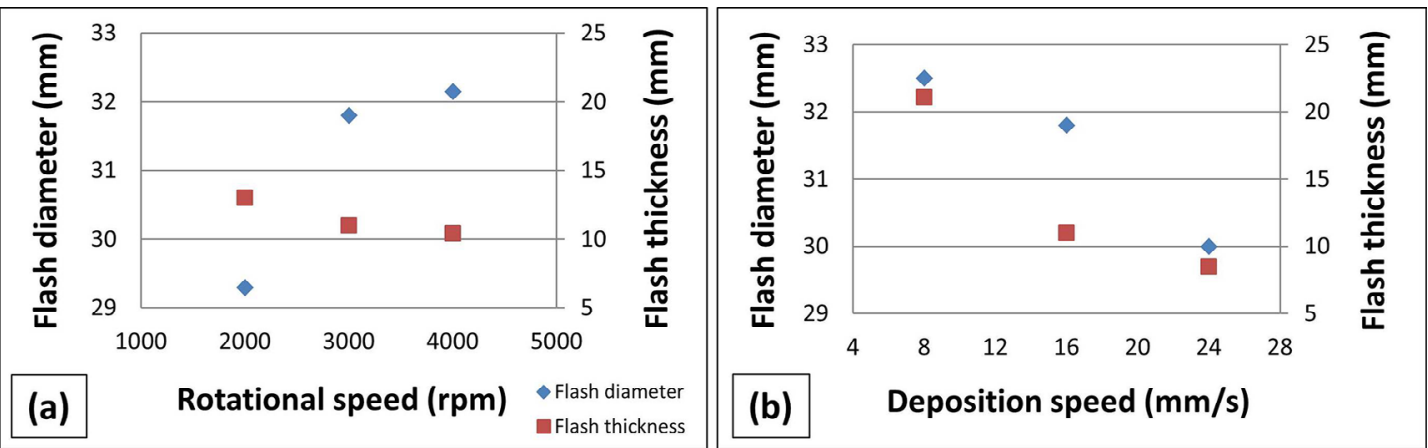

Figure 4. Effect of (a) rotational and (b) deposition speeds on the flash formed on the rod. 
The slight reduction in thickness is related to the considered increase in curvature of the flash.

The consumption rate relates to the speed at which the rod material is consumed through plastification, deposition and flash formation. Since the consumption rate was constant at this study, as lower the deposition speed, more material is plasticized, and flash' diameter and thickness are higher. Therefore, by increasing the deposition speed, both flash' diameter and thickness ascending on the rod decreases, being a lower amount of material that passes on to the flash and, consequently, more material can be deposited and higher is the deposition efficiency (Fig. 3). Nevertheless, the values of flash characteristics and deposition efficiency do not directly correlate with the deposit width or thickness ${ }^{4,9}$.

\subsection{Microstructure and hardness of the rods}

The FS process imposes a severe plastic shear deformation and thermal cycle, changing the original microstructure of the consumable rod and leading to recrystallization, grain refinement and, depending on the processed material, phase transitions $\mathbf{s}^{1,15}$.
The microstructure of the rod base material of Ti-6Al$4 \mathrm{~V}$ is formed by two phases, presenting an alpha-beta $(\alpha+\beta)$ structure at room temperature, which exhibits equiaxial alpha and intergranular beta grains. Fig. 5 shows the change in microstructure at the rod tip due to the higher temperatures achieved, generated by the hot work at the rod tip and the coating generated heat that is conducted along the consumable rod, pre-heating the material and allowing its plastic deformation ${ }^{8}$. Therefore, the heat conducted to the rod is not considered as a loss of energy, since pre-heats the consumable material that will be deposited as a coatings.

As can be noticed in Fig. 5 (a) there is a transition zone between the base material of the rod and the HAZ of the rod, due to the considerable increase in the temperature due to the diffusion. The employment of high temperatures on base material lead to grain growth ${ }^{8}$, and consequently it can be seen by Fig. 5 (b) and (c) the microstructure evolution at the rod tip where occurred the transition to acicular alpha grains after cooling, and an increase in prior beta grains until the rod tip.

Fig. 6 presents the microhardness evolution on coating and consumable rod, perpendicularly to the interface.
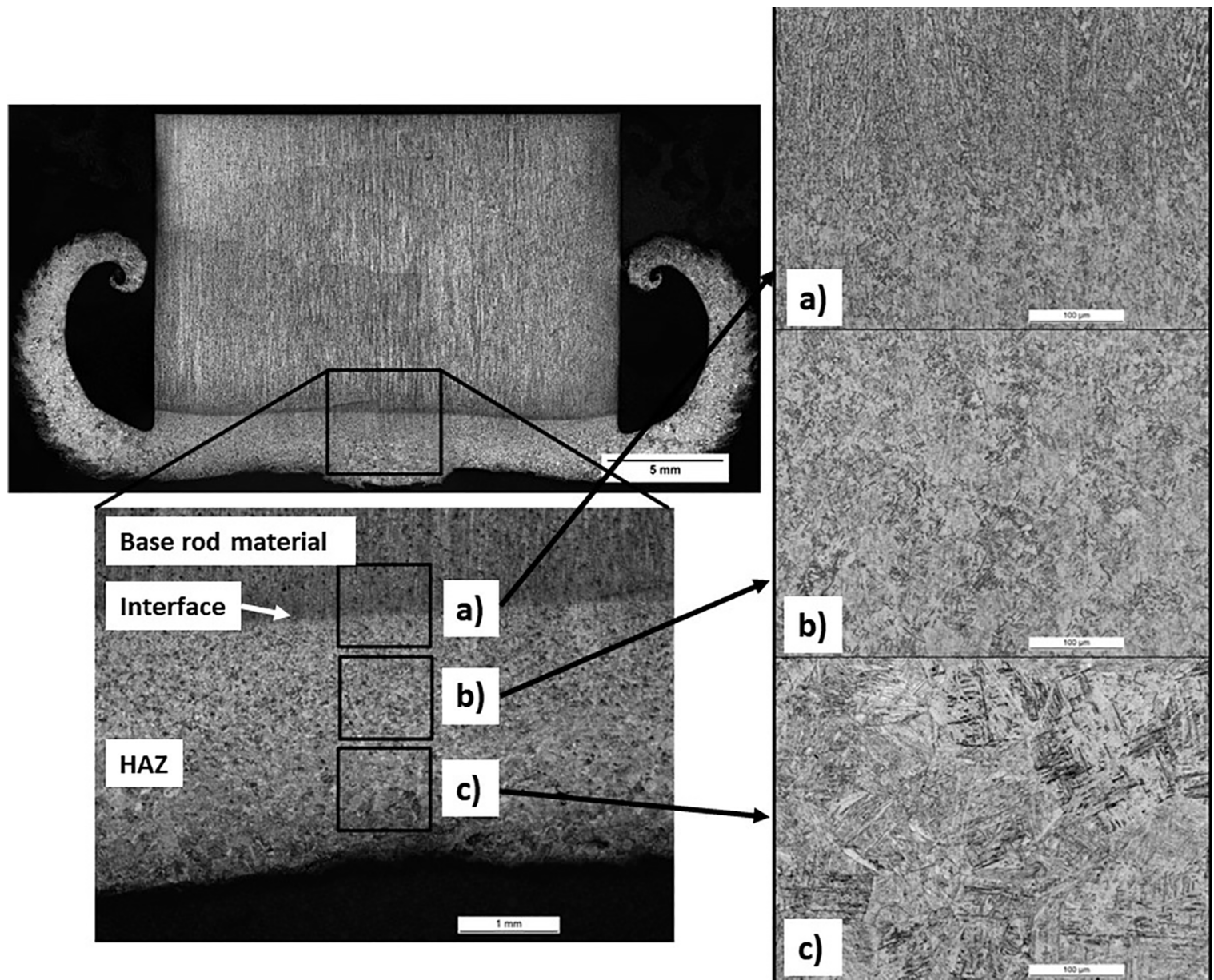

Figure 5. Microstructural evolution of the consumable rod: (a) transition zone between the base material of the rod and the HAZ of the rod; (b) acicular alpha grains after cooling and (c) prior beta grains until the rod tip. 


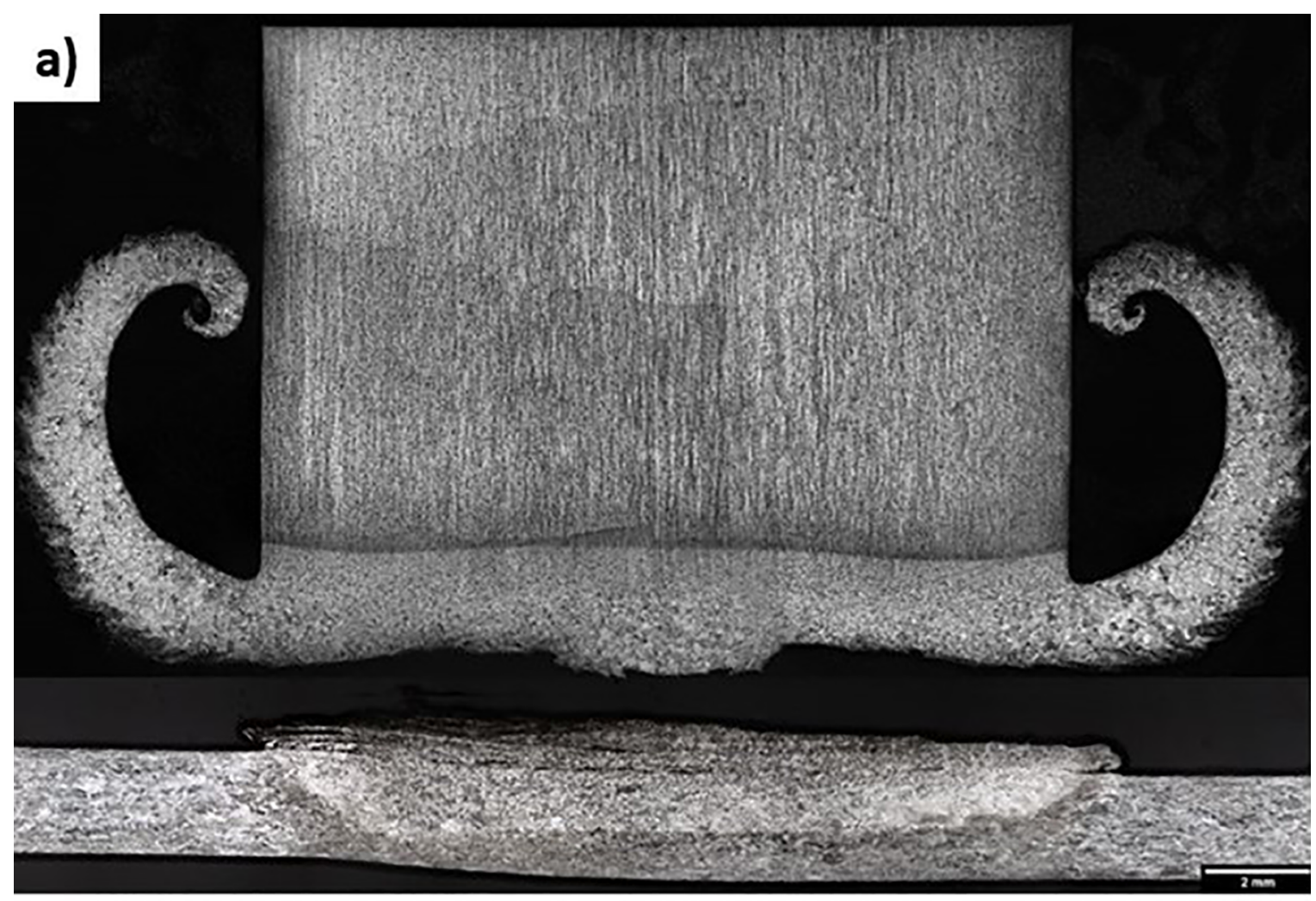

b)

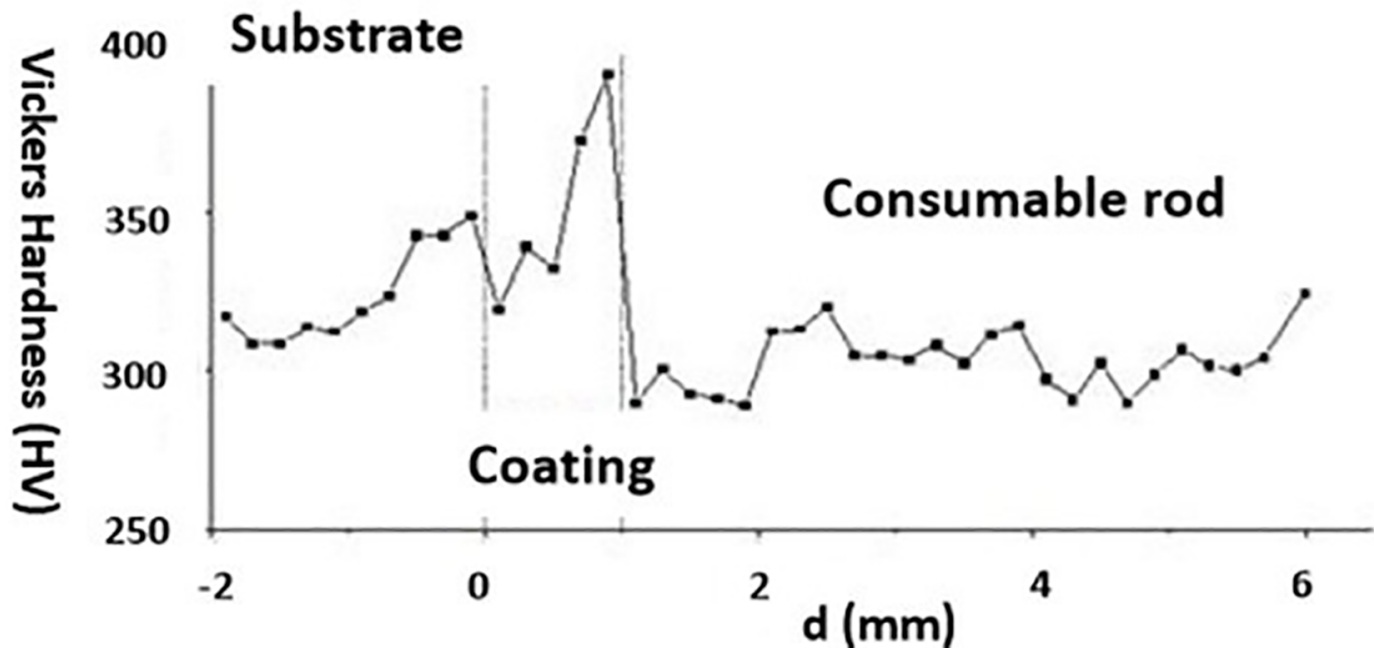

Figure 6. (a) Macrographs of the cross sections of rod and deposited coating on substrate and (b) microhardness evolution along the macrograph.

Hardness increases along the substrate, being higher in the heat affected zone (HAZ), and increases along the coating, presenting the highest value near the coating surface. In the rod, a lower hardness is noticed close to the substrate, due to an increase in its grains size, as can be seen in Fig. 5(c), and then presented close values for the rest of the rod, which are close to the original base material (300 HV).

\section{Conclusions}

From the work done, the following conclusions can be drawn. The flash formation and consequently the deposition efficiency is dependent on the process parameters:

- Increasing deposition speed leads to a decrease in thickness and diamater of the flash ascending on the consumable rod, increasing the deposition efficiency; 
- $\quad$ increasing rotational speed, leads to an increase in flash diameter but reduction in thickness due to the centrifugal forces applied.

- No correlation could be performed between deposition efficiency and rotational speed.

- Microstructural evolution could be seen on the rod, with an increase in grain size next to the rod tip, reducing the hardness.

\section{Acknowledgments}

The authors would like to acknowledge the UFPE laboratory (COMPOLAB), CNPq and CAPES, for giving financial support and all the conditions to carry out this work.

\section{References}

1. Fitseva V, Krohn H, Hanke S, Santos JF, et al. Friction surfacing of Ti-6Al-4V: process characteristics and deposition behaviour at various rotational speeds. Surface and Coatings Technology. 2015;278:56-63.

2. Fukakusa K. On the characteristics of the rotational contact plane - a fundamental study of friction surfacing. Welding International. 1996;10(7):524-529.

3. Hanke S. Microstructural alterations of commercial metallic alloys by friction surfacing [thesis]. Duisburg, Essen: University of Duisburg-Essen; 2014.

4. Hanke S, Santos JF. Comparative study of severe plastic deformation at elevated temperatures of two aluminium alloys during friction surfacing. Journal of Materials Processing Technology. 2017;247:257-267.

5. Gandra J, Miranda RM, Vilaça P. Performance analysis of friction surfacing. Journal of Materials Processing Technology. 2012;212(8):1676-1686.
6. Li J, Shinoda T. Underwater friction surfacing. Surface Engineering. 2000;16(1):31-35.

7. Vale N, Fitseva V, Hanke S, Urtiga Filho S, Santos JF. Influence of rotational speed in the friction surfacing of titanium grade 1 on Ti-6Al-4V. Materials Research. 2017;20(Suppl 2):830-835.

8. Fitseva V, Hanke S, Santos JF, Stemmer P, Gleising B. The role of process temperature and rotational speed in the microstructure evolution of Ti-6Al-4V friction surfacing coatings. Materials and Design. 2016;110:112-123.

9. Vale N, Urtiga Filho S, Fitseva V, Hanke S, Santos JF. Revestimento depositado por atrito de Ti-6Al-4V: efeitos da velocidade de deposição. In: IX Congresso Nacional de Engenharia Mecânica; 2016 ago 21-25; Fortaleza, Ceará, Brasil. Fortaleza (CE): ABCM; 2016.

10. Hanke S, Staron P, Fischer T, Fitseva V, Santos JF. A method for the in-situ study of solid-state joining techniques using synchrotron radiation - observation of phase transformations in Ti-6Al-4V after friction surfacing. Surface and Coatings Technology. 2018;335:355-367.

11. Gandra J, Pereira D, Miranda RM, Vilaça P. Influence of process parameters in the friction surfacing of AA $6082-\mathrm{T} 6$ over AA 2024-T3. Procedia CIRP. 2013;7:341-346.

12. Barnes S, Bhatti A, Steuwer A, Johnson R, Altenkirch J, Withers P. Friction stir welding in HSLA-65 steel: Part I. Influence of weld speed and tool material on microstructural development. Metallurgical and Materials Transactions: A. 2012;43(7):2342-2355.

13. Gandra J, Krohn H, Miranda RM, Vilaça P, Quintino L, Santos JF. Friction surfacing - a review. Journal of Materials Processing Technology. 2014;214(12):1062-1093.

14. Rafi HK, Ram GDJ, Phanikumar G, Rao KP. Microstructural evolution during friction surfacing of tool steel H13. Materials and Design. 2011;32:82-87.

15. Hanke S, Beyer M, Silvonen A, Santos JF, Fisher A. Cavitation erosion of Cr60Ni40 coatings generated by friction surfacing. Wear. 2013;301:415-423. 